\title{
Fibre optic track vibration monitoring system
}

\section{Stanislav Kepak ${ }^{1}$, Jakub Cubik ${ }^{1}$, Petr Zavodny ${ }^{1}$, Petr Siska ${ }^{1}$, Alan Davidson ${ }^{2}$, Ivan Glesk $^{2}$ and Vladimir Vasinek ${ }^{1}$}

1 Department of Telecommunications, Faculty of Electrical Engineering and Computer Science, VSBTechnical University of Ostrava, 17. listopadu 15, 70833 Ostrava-Poruba, Czech Republic

2 Department of Electronic and Electrical Engineering, Faculty of Engineering, University of Strathclyde, 204 George Street, Glasgow, G1 1XW, United Kingdom

E-mail: stanislav.kepak@,vsb.cz; Phone: +420 597321664

\begin{abstract}
Track monitoring systems are fundamental part of railroads. With the electrification and development of train traction control electronics, levels of electromagnetic interference (EMI) close to railroad monitoring systems is reaching critical levels. Unreliable track safety and fault monitoring systems could affect the efficient and safe functioning of railroads and therefore strict demands must be placed on track monitoring systems to ensure electromagnetic compatibility (EMC). The Prague subway system is not an exception. An alternative track vibration monitoring system has been proposed, demonstrated and tested along a single railway track in the Prague subway system. Two passive detection systems placed $50 \mathrm{~m}$ and $1.3 \mathrm{~km}$ away from the control room were used to measure tunnel vibrations triggered by passing trains free from the effect of any unrelated EMI existing in the subway tunnel. Fibre optic based systems immune to EMI can offer an efficient solution to both track and train monitoring.
\end{abstract}

Keywords vibration measurements, track monitoring, fibre optic sensor, EMI-free

Acknowledgments The research described in this article could be carried out thanks to the active support of the Ministry of Education, Youth and Sports of the Czech Republic through grant project no.

CZ.1.07/2.3.00/20.0217 within the frame of the operation programme Education for competitiveness financed by the European Structural Funds and from the state budget of the Czech Republic. This article was also supported by project Technology Agency of the Czech Republic TA03020439 and TA04021263. The research has been partially supported by the projects no. SP2016/149 and VI20152020008. 


\section{Introduction}

Track monitoring systems are important for the safe and efficient operation of railway systems. In recent years new safety standards have been introduced in line with the expansion of high-speed train services. Monitoring systems help to maintain the safety of railway tracks and trains by monitoring and warning of potentially hazardous track conditions. Such systems include velocity monitors, derailment sensors, track integrity sensors and track loading sensors to name a few.

At present most of these monitoring systems work entirely on electrical or electro-magnetic principles (Parker 2012). Since in electrified systems track rails are often used to carry the traction return current, the generation of electromagnetic interference (EMI) is almost inevitable. Even with well safeguarded circuits, these systems are still subject to operational failures. These systems must therefore be backed up with other systems, preferably operating on a different principle.

Fibre optic systems offer an EMI-free alternative to conventional systems. The simplest fibre optic systems are based on the macro or micro bending effect (Sokoiowski et al. 2004). Such a sensor, placed between rail and its sleeper, can provide information on train direction, speed and even weight. Magneto-optic systems use the Faraday Effect to optically detect alterations in the magnetic field of a permanent magnet located near to the sensor (Veeser et el. 1998). As a result of the influence on the field by the wheel of a railway vehicle, a variation in the intensity of optical radiation passing through the sensor occurs. Fibre Bragg grating (FBG) systems are capable of monitoring track conditions as well as collecting train axle, velocity and overall weight information (Yuen 2014; Tam et al. 2011). The operating principle is based on measuring strain changes in the rail caused by the passage of trains at the measurement points using a FBG detector installed on the rail.

Distributed fibre optic monitoring systems are capable of delivering near real-time information about rail track deformation induced by the passage of a train (Peng et el. 2014; Minardo et el. 2013). These systems use optical-time-domain reflectometry to interrogate the optical sensing cable buried along the track or attached to the rails. The major advantage is in the capability of continuous track monitoring at lengths of over $100 \mathrm{~km}$.

Interferometric monitoring systems have proven to be applicable to railways too, but mostly for current monitoring (Kumagai et al. 2013). These systems are very sensitive due to their principle of operation. The new fibre optic detection system (FODS) presented is based on a Mach-Zehnder interferometer (MZI) and has been designed to be robust and reliable for use in the harsh environment of the subway. The system demonstrated is capable of capturing track vibration caused by the passage of trains and was developed as a back-up to conventional systems mainly because of its resistance to EMI. Unlike conventional approaches such as piezoelectric and capacitive accelerometers newly developed system was successfully tested on the Prague subway.

\section{Experimental setup}

The FODS comprises an MZI fibre optic sensor which is formed by two $1 \times 2$ power couplers with a $50 \%$ splitting ratio and a two $2 \mathrm{~m}$ long single mode $3 \mathrm{~mm}$ jacketed optical fibres, see Fig. 1a. The measuring fibre is encased in polystyrene vibration isolation material (Kepak et al. 2013), Fig. 1b, while the reference fibre is attached to a resonance glass plate (Cubik et al. 2013), Fig. 1c. A protective plastic casing surrounds the whole sensor assembly, see Fig. 1d. The detection system input and output was connectorised with FC/APC connectors shown as 1 and 2 in Fig. 1.

The overall arrangement for the monitoring system using the devolved FODS is shown in Fig. 2. A 0dBm DFB laser operating at a wavelength of $1550 \mathrm{~nm}$ with $1 \mathrm{GHz}$ linewidth was selected for use as the light source. The laser has built-in current and temperature controllers and default setting values were used to maintain stable operating conditions.

A Thorlabs PDA10CS trans-impedance amplified photodetector was used for signal detection (shown as PIN in Fig. 2). The signal from the photodetector was then fed through a passive bandpass filter (BPF in Fig. 2) with a passband of $8 \mathrm{~Hz}$ to $20 \mathrm{kHz}$ to eliminate any aliasing and to block the DC. The signal was then sampled and quantized using a National Instruments USB-6210 measuring card (A/D in Fig. 2) and the LabVIEW development suite was used to develop the GUI for FODS and for signal recording and processing. The received signal was processed in both time and frequency domains. 


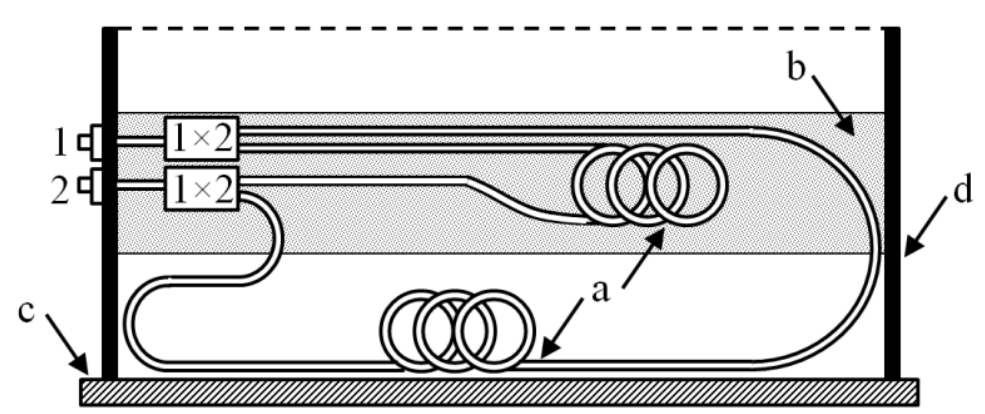

Fig. 1 Fibre optic detection system side view

$a$ Sensing and reference fibre arm of the MZI sensor

$b$ Reference fibre arm insulating material

$c$ Glass resonance plate

$d$ Plastic enclosure

1, 2 FC/APC connectors

The DFB laser and detector system seen in Fig. 2a was placed in the control room at the nearest subway station. The laser output was fed into the FODS via a Minicord Duplex cable shown in Fig. 2b. The FODS was connected to both cable ends using FC/APC connectors 1 and 2 (see Fig. 2c), while the second fibre in the cable assembly (see b in Fig. 2) was used to transmit the signal from FODS back to the subway station control room. Depending on the measurement, the FODS (Fig. 2c) was firmly attached either to the subway tunnel floor or to the safety walk.

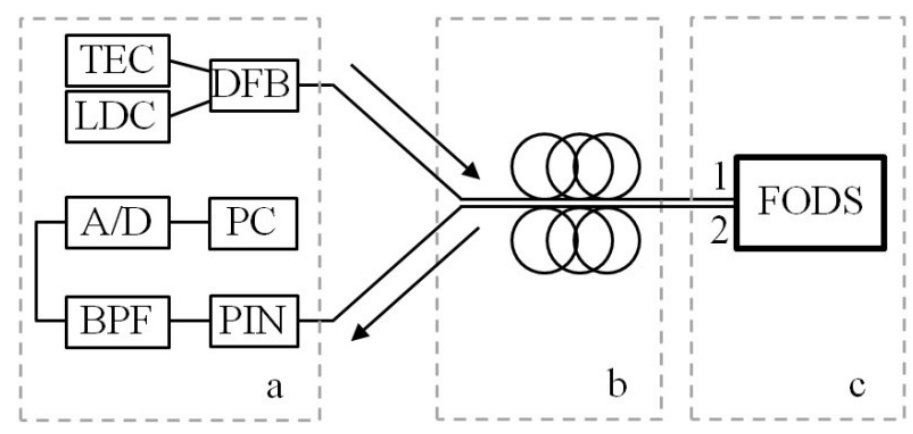

Fig. 2 Vibration monitoring system configuration with one sensor

$a$ Processing unit

$b$ Fibre link to and from the detection system

$c$ Fibre optic detection system

The system was tested in two locations. The first measurement location was near the subway station platform (see FODS-1 in Fig. 3) and the sensor was connected to the control room via a 50m long optical duplex cable. FODS-1 was firmly mounted on the tunnel floor at the tunnel side near the rail track point. The second detection system was in the tunnel (FODS-2 seen in Fig. 3) and was placed on the safety walk inside the tunnel and connected to the control room via a $1.3 \mathrm{~km}$ long optical duplex cable. 


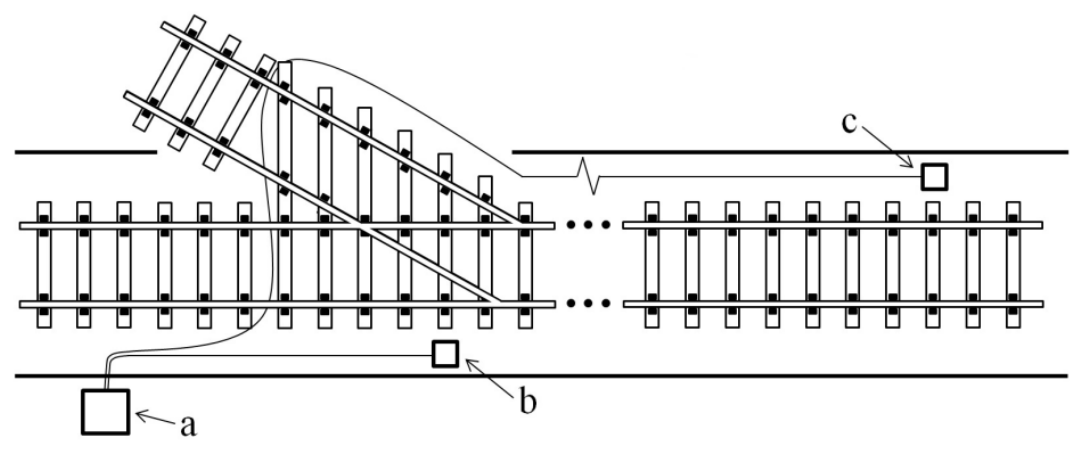

Fig. 3 Detection system placement in the tunnel

$a$ Processing unit in Control room

$b$ FODS-1

$c$ FODS-2

\section{Detection system operating principles}

The deployed FODS uses a balanced Mach-Zehnder fibre optic interferometer operating on the principle of phase modulation. The optical phase delay $\phi$ of light passing through an optical fiber is given by:

$$
\phi=\mathrm{kn} \mathrm{L}
$$

Where $n$ is the refractive index of the fibre core, $k$ is optical wavenumber in vacuum $(\mathrm{k}=2 \pi / \lambda, \lambda$ is light source wavelength) and $L$ is the physical length of the fibre. The product $n L$ represents the optical path length. For variations in the phase delay of the interferometer, we can write:

$$
\frac{d \phi}{\phi}=\frac{d \mathrm{k}}{\mathrm{k}}+\frac{d \mathrm{n}}{\mathrm{n}}+\frac{d \mathrm{~L}}{\mathrm{~L}}
$$

When supply current and temperature are stabilized, then $k$ can be considered to be constant since it depends only on the wavelength of the source. The phase change is therefore proportional to $n$ and $L$. Changes in pressure, temperature or magnetic field are processes that lead to different contributions to $d \phi$ via the $d \mathrm{~L}$ and $d \mathrm{n}$ terms. Changes in $d \mathrm{~L}$ are due to changes in core and cladding material (Giallorenzi et el. 1982), while changes in the refractive index are due to changes in the optical stress coefficient (Butter et el. 1978).

The FODS is compact and uses two couplers with coupling coefficients $c_{1}$ and $c_{2}$. Optical loss in measuring and reference arm are denoted by the coefficients $\alpha_{s}$ and $\alpha_{r}$. The output intensity of the FODS can be expressed as (Udd et el. 2011):

$$
\mathrm{I}=\mathrm{I}_{0}\left[\alpha_{\mathrm{r}} \mathrm{c}_{1} \mathrm{c}_{2}+\alpha_{\mathrm{s}}\left(1-\mathrm{c}_{1}\right)\left(1-\mathrm{c}_{2}\right)+2 \sqrt{\alpha_{\mathrm{r}} \alpha_{\mathrm{s}} \mathrm{c}_{1} \mathrm{c}_{2}\left(1-\mathrm{c}_{1}\right)\left(1-\mathrm{c}_{2}\right) \cos \left(\phi_{\mathrm{r}}-\phi_{\mathrm{s}}\right)}\right]
$$

Where $\phi_{r}$ is the phase shift in reference arm and $\phi_{s}$ is the phase shift in measuring arm.

For simplification of (3) is assumed that the polarization state of the light in both arms is the same, light source coherence length is within the interferometer path length difference $\Delta L$, also is expected that $\alpha=\alpha_{\mathrm{r}}=\alpha_{\mathrm{s}}$ (so that transmission losses in both arms of the interferometer are equal) and the coupling coefficients are $c_{1}=$ $\mathrm{c}_{2}=0,5$, so that the fringe visibility is $\mathrm{V}=1$. The intensity at the FODS output can then be rewritten as:

$$
\mathrm{I}=\frac{\mathrm{I}_{0} \alpha}{2}(1+\cos \Delta \phi)
$$

Where $I_{0}$ is light intensity on the input of the first coupler and $\Delta \phi=\left(\phi_{\mathrm{r}}-\phi_{\mathrm{s}}\right)$ is the phase difference between both arms of the interferometer. 
Neglecting transmission losses on the fibre link, the detector output current (photocurrent) resulting from illumination by the light intensity is given by:

$$
\mathrm{i}=\varepsilon \mathrm{I}_{0} \alpha \cos \left(\phi_{\mathrm{d}}+\phi_{\mathrm{s}} \sin \omega \mathrm{t}\right)
$$

Where $\varepsilon$ is the responsivity of the photodetector $\left(1.05 \mathrm{~A} . \mathrm{W}^{-1}\right.$ at $\left.1550 \mathrm{~nm}\right)$. The phase difference $\Delta \phi$ is separated into the signal term of amplitude $\phi_{s}$ varying with frequency $\omega$ and phase shift $\phi_{d}$. Signal term of phase difference was retrieved in developed application.

The photodetector converts the input photocurrent $i(t)$ to the output voltage $u(t)$. The output voltage signal $u(t)$ is passed through an anti-aliasing filter $(20 \mathrm{kHz}$ low-pass filter) to limit the signal bandwidth. The filtered signal is then sampled by a measuring card and converted to a set of uniformly spaced samples $x[n]$. A sampling rate $f_{s} 40 \mathrm{kS} / \mathrm{s}$ was chosen to allow signals up to frequency $20 \mathrm{kHz}$ to be captured. These samples were then stored in a data log file.

\section{Experimental results analysis}

In addition to vibration measurements which were the subject of the original contract for Prague subway, the measured results were also analysed in such way that train operations can be monitored. These measurements were performed using two FODS operating simultaneously. During a 5 hour period the detection system recorded 64 trains in total which was in line with the subway timetable. However, they also recorded two types of false events. The first were caused by 'after echoes' related to trains that have passed by and the second were caused by random noise in the tunnel. The after echoes were eliminated by introducing guard-time intervals. For FODS-1 the guard-time interval of $15 \mathrm{~s}$ was found to be sufficient. However for FODS-2 a guard interval of up to 50 s was required to achieve the best results, see Table 1 .

Table 1: The results analysis of trains passing near the sensors

\begin{tabular}{llllll}
\hline total no. of trains & FODS location & guard interval & correctly detected & missed trains & false alarms \\
\hline 64 & station & $15 \mathrm{~s}$ & 64 & 0 & 0 \\
64 & tunnel & $30 \mathrm{~s}$ & 64 & 0 & 8 \\
64 & tunnel & $40 \mathrm{~s}$ & 64 & 0 & 4 \\
64 & tunnel & $45 \mathrm{~s}$ & 64 & 0 & 1 \\
64 & tunnel & $50 \mathrm{~s}$ & 64 & 0 & 0 \\
\hline
\end{tabular}

In order to eliminate the tunnel noise interference, the measured signal was converted from time to frequency domain by using a discrete Fourier transform (DFT) by using Eq. 6.

$$
X_{k}=\sum_{n=0}^{N-1} x_{n} \cdot\left(\cos \left(-2 \pi k \frac{n}{N}\right)+j \sin \left(-2 \pi k \frac{n}{N}\right)\right), k \in \mathbb{Z}
$$

Where $x_{n}$ is a set of uniformly spaced samples of the measured time domain signal acquired during the passage of a train, $N$ is a total number of samples and $k$ is a positive integer. The resulting $X_{k}$ represents the amplitude of the $k^{\text {th }}$ harmonic frequency. To achieve the required spectral resolution and bandwidth, the zoom spectrum algorithm was used instead of the DFT. The zoom spectral algorithm parameters were: frequency filtering from 0 to $1 \mathrm{kHz}$ using a low pass Finite Impulse Response (FIR) filter with a cut-off frequency of $1 \mathrm{kHz}$ and signal down sampling (decimation) was applied to achieve a spectral resolution of 1000 lines. To obtain the amplitude spectrum the resulting signal was then subjected to a Fast Fourier Transformation (FFT). The FFT was applied to 10 consecutive sections of the time domain signal and the average of these spectra was calculated using RMS averaging and a linear weighting. A Hanning window function ( $0 \%$ overlap) was applied to each time domain section. Bandwidth reduction to $1 \mathrm{kHz}$ was performed due to spectral properties of railway noise (Thompson 2009). 
Fig. 4 is an example of the frequency spectrum of a false alarm recorded in the tunnel. Fig. 5 shows the vibration signal (rolling noise) from a passing train recorded by the FODS-2 and Fig. 6 is its frequency spectrum calculated using the methodology described above. By comparing Fig. 4 and 6 the spectral region corresponding to the false alarm can be easily noted and the desired signal identifying the passing train can be then be obtained. Similarly, FODS-1 was used for the identification of passing by trains in the different tunnel location leading similar positive results.

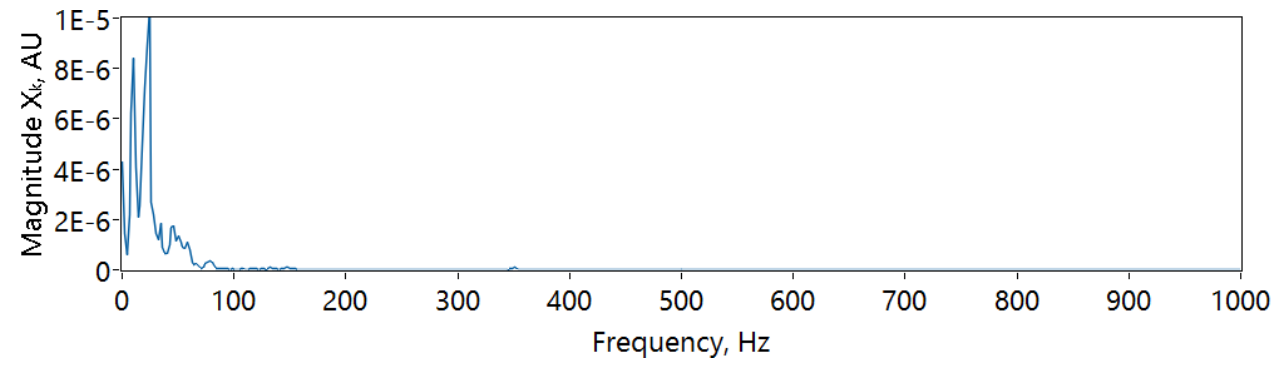

Fig. 4 Frequency response of a false alarm in the tunnel

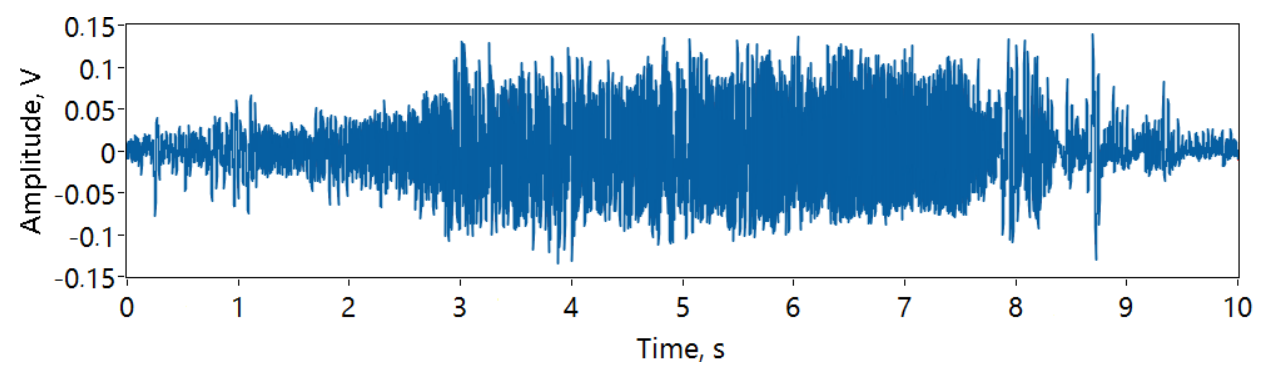

Fig. 5 Signal from a passing by train recorded by sensor FODS-2

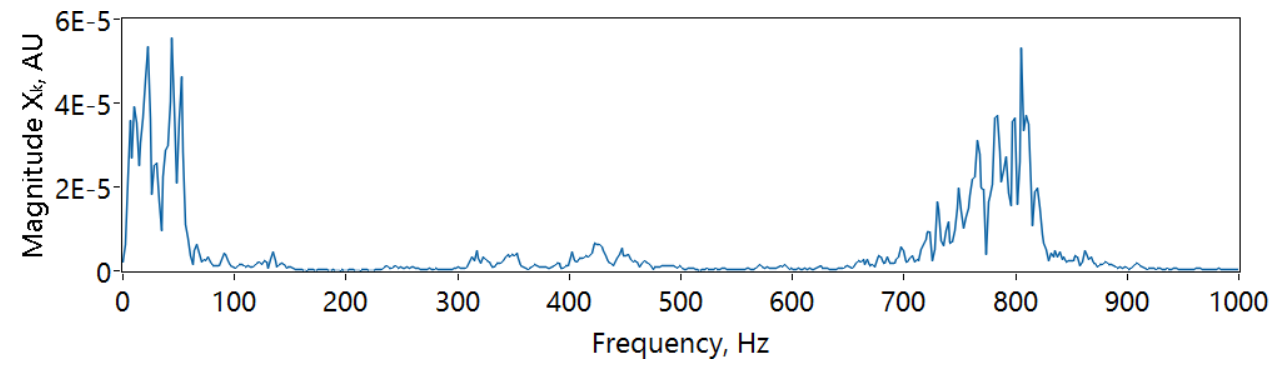

Fig. 6 Calculated frequency response of a passing by train

Using multiple suitably located FODS, train operations could be monitored helping to support subway operational safety. As a result of reliable train detection a day-to-day traffic analysis can be performed. In Fig. 7 the measured punctuality for one day traffic for FODS-1 can be seen. The data was collected as timestamps and these values were then compared with the timetable and the resulting time differences were plotted (positive values means trains are delayed). This data could provide an overall picture of subway traffic through the course of the day. 


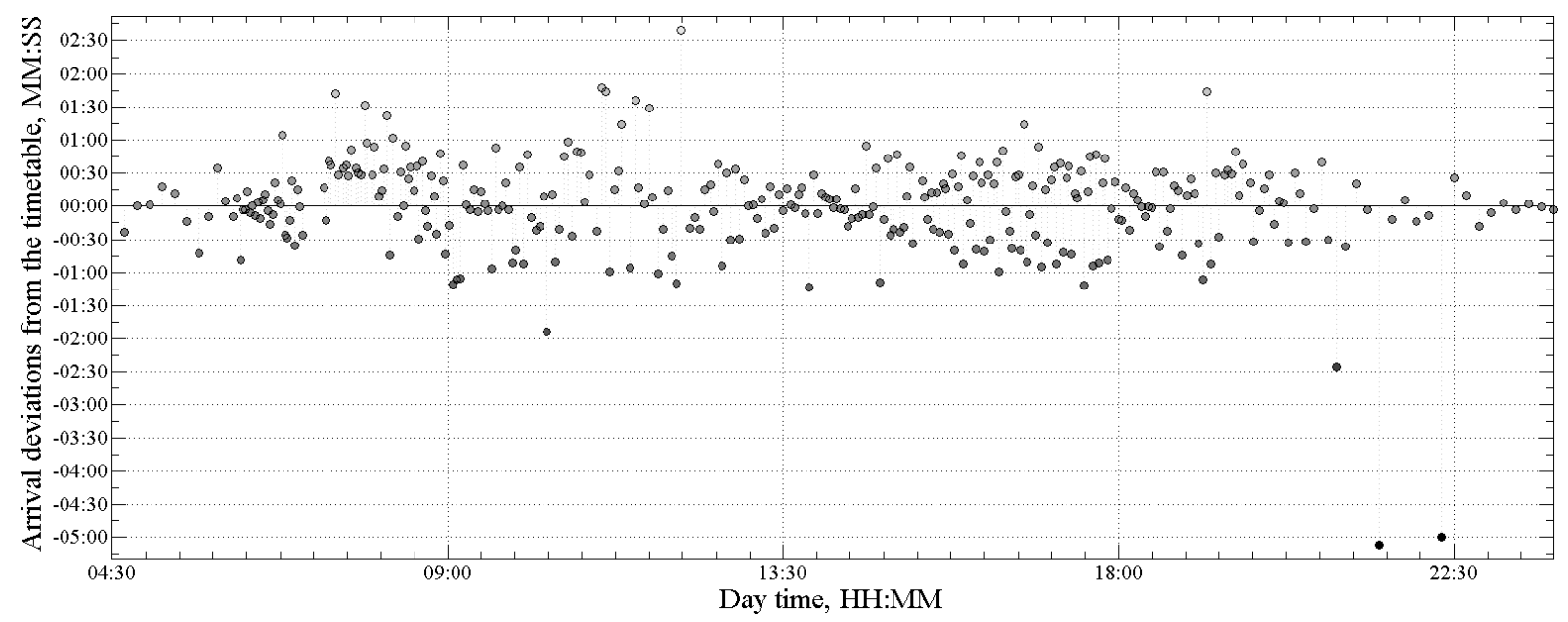

Fig. 7 Measured trains arrival deviations from the timetable in a given day

Since the FODS-1 was located near a track point, impact noise measurements were possible. Impact noise appears when discontinuities in the rail surface such as rail joints, welds, points or crossings occurs. Its amplitude is higher than other noises such as rolling noise (Parker 2012) and depends on variables such as joint geometry, train weight, velocity and wheel shape and condition (Vér et el. 1976). With the monitoring of impact noise, it is possible to monitor/count train axles. The dependence of impact noise on train velocity should be linear, thus allowing the monitoring of trains at critical points.

An example of measured impact noise at the track point is given in Fig. 8. The respective peaks seen in Fig. 8 correspond to the impact noise generated by individual train axles crossing the track point. The analysis for this measurement is given in Table 2. The speed was calculated from the known carriage central pivot point's distance and timestamps of axle impacts. The noise amplitude was measured as total noise amplitude when the bogies passed the track point and the impact noise amplitude was obtained after deduction of other noise present such as rolling noise. Considering similar wheel condition and carriage weight, an approximate linear model could be constructed to estimate the train velocity based on the impact noise measurements. Also, with knowledge of the exact speed, impact noise dependent variables can be calculated.

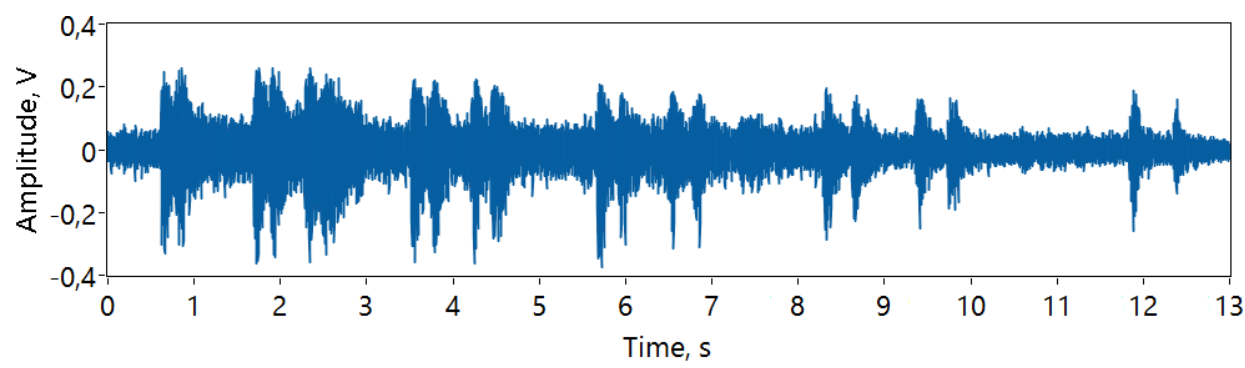

Fig. 8 Signal from a passing by train recorded by sensor FODS-1

Table 2: Impact noise measurement summary

\begin{tabular}{llll}
\hline carriage & measured speed $\left[\mathrm{km} \cdot \mathrm{h}^{-1}\right]$ & noise amplitude $[\mathrm{AU}]$ & impact noise amplitude [AU] \\
\hline 1 & 42 & 1 & 1 \\
2 & 37,5 & 0,96 & 0,95 \\
3 & 30,9 & 0,88 & 0,86 \\
4 & 25,3 & 0,79 & 0,74 \\
5 & 18,5 & 0,62 & 0,54 \\
\hline
\end{tabular}




\section{Conclusions}

Track vibration interferometric sensor systems have the potential for a wide range of applications including the monitoring of train operations such as train punctuality or velocity. The designed system presented in this article was able to function flawlessly in continuous operation in the Prague subway network and will be deployed as a backup to the existing conventional approach. Further research will be aimed at processing algorithms and hardware to interpret the measured results in near real-time.

\section{References}

1. Parker, S.: 'Track Design Handbook for Light Rail Transit' (Transportation Research Board, Washington, MD, USA, 2012, second edition)

2. Sokoiowski, K., Domanski, A.W., Wolinski, T.R.: 'Intensity-based fiber optic bend sensor for potential railway application', Proceedings of SPIE - The International Society for Optical Engineering., 2004, vol. 5484, pp. 417-420, doi 10.1117/12.568927

3. Veeser, L.R., Forman, P.R., Rodriguez, P.J.: 'Lensless Magneto-Optic Speed Sensor', United States Patent, 1998

4. Yuen, K.K.: 'Novel application of a fibre optic-based train weigh-in-motion system in railway', HKIE

Transactions Hong Kong Institution of Engineers, 2014, vol. 21, no. 4, pp. 272-280, doi 10.1080/1023697X.2014.970752

5. Tam, H., Liu, S., Ho, S., Ho, T.: 'Fiber bragg grating sensors for railway systems' in Cusano, A., Cutolo, A., Albert, J.: 'Fiber bragg grating sensors: Recent advancements, industrial applications and market exploitation' (Bentham Science Publishers, Sharjah, 2011), doi 10.2174/978160805084011101010197

6. Peng, F., Duan, N., Rao, Y., Li, J.: 2014, 'Real-time position and speed monitoring of trains using phasesensitive OTDR', IEEE Photonics Technology Letters, 2014, vol. 26, no. 20, pp. 2055-2057, doi 10.1109/LPT.2014.2346760

7. Minardo, A., Porcaro, G., Giannetta, D., Bernini, R., Zeni, L.: 'Railway traffic monitoring using Brillouin distributed sensors', Proceedings of SPIE - The International Society for Optical Engineering, 2013, vol. 8794, doi 10.1117/12.2025991

8. Kumagai, T., Ohnuki, W., Hayashiya, H., Nishida, K.: 'Interferometric fiber-optic electric current sensor for railway power systems', IEEJ Transactions on Sensors and Micromachines, 2013, vol. 133, no. 2, pp. 42-47, doi 10.1541/ieejsms. 133.42

9. Kepak, S., Cubik, J., Doricak, J., Vasinek, V., Siska, P., Liner, A., Papes, M.: 'The arms arrangement influence on the sensitivity of Mach-Zehnder fiber optic interferometer', Proceedings of SPIE - The International Society for Optical Engineering, 2013, vol. 8774, doi 10.1117/12.2017305

10. Cubik, J., Kepak, S., Doricak, J., Vasinek, V., Jaros, J., Liner, A., Papes, M., Fajkus, M.: 'The usability analysis of different standard single-mode optical fibers and its installation methods for the interferometric measurements', Advances in Electrical and Electronic Engineering, 2013, vol. 11, no. 6, pp. 535-542, doi 10.15598/aeee.v11i6.885

11. Giallorenzi, T., Bucaro, J., Dandridge, A., Sigel, G., Cole, J., Rashleigh, S., Priest, R.: 'Optical fiber sensor technology', IEEE Journal of Quantum Electronics, 1982, vol. 18, no. 4, pp. 626-665, doi

10.1109/JQE.1982.1071566

12. Butter, C.D., Hocker, G.B.: 'Fiber optics strain gauge', Applied Optics, 1978, vol. 17, no. 18, pp. 2867-2869, doi 10.1364/AO.17.002867

13. Udd, E., Spillman, W.B.: 'Fiber Optic Sensors: An Introduction for Engineers and Scientists: Second Edition' (John Wiley \& Sons, New Jersey, 2011), doi 10.1002/9781118014103

14. Thompson, D.: 'Railway Noise and Vibration: Mechanisms, Modelling and Means of Control' (Elsevier, Amsterdam, 2009)

15. Vér, I. L., Ventres, C. S., Myles, M. M.: 'Wheel/rail noise-part III: Impact noise generation by wheel and rail discontinuities', Journal of Sound and Vibration, 1976, vol. 46, no. 3, pp. 395-417, doi 10.1016/0022460X(76)90863-4(1976). 